\title{
Anti-Vascular Endothelial Growth Factors as a Potential Risk for Implant Failure: A Clinical Report
}

\author{
Elham Emami $\mathbb{D}^{1},{ }^{1}$ Pierre de Grandmont, ${ }^{2}$ Mélanie Menassa, ${ }^{2}$ Nicholas Audy, ${ }^{3}$ \\ and Robert Durand $\mathbb{( i D}^{2}$ \\ ${ }^{1}$ Faculty of Dentistry, McGill University, Montreal, QC, Canada \\ ${ }^{2}$ Faculty of Dentistry, Université de Montréal, Montreal, QC, Canada \\ ${ }^{3}$ Private Clinic, Saint-Jérôme, QC, Canada \\ Correspondence should be addressed to Elham Emami; elham.emami@mcgill.ca
}

Received 20 January 2019; Revised 29 October 2019; Accepted 14 January 2020; Published 3 February 2020

Academic Editor: Indraneel Bhattacharyya

Copyright $(92020$ Elham Emami et al. This is an open access article distributed under the Creative Commons Attribution License, which permits unrestricted use, distribution, and reproduction in any medium, provided the original work is properly cited.

Knowledge of the risk factors for implant osseointegration is essential for clinical decision-making and optimizing treatment success. This clinical report presents a rare case of implant failure in a patient who received intravitreal injections of a vascular endothelial growth factor (VEGF) inhibitor for the treatment of age-related macular degeneration. Following CARE guidelines, the report presents a case rehabilitated with a mandibular 2-implant overdenture using the immediate-loading protocol and standard procedures. The implants failed within six weeks of immediate loading although primary stability $(\geq 50 \mathrm{Ncm})$ was achieved during surgery and clinical follow-ups did not show any deviance from standard implant care or patient-related complications. Further investigation suggested that the intake of a VEGF inhibitor may be the cause of failure. This clinical report highlights the importance of systemic risk factors in implant success and their consideration during planning for implant-assisted treatment.

\section{Introduction}

Osseointegration is defined as the "process whereby clinically asymptomatic rigid fixation of alloplastic materials is achieved and maintained in bone during functional loading" [1]. A predictable outcome of any bone interaction is dependent on the maintenance of living status of bone. Therefore, angiogenesis, which is the outgrowth of new capillary blood vessels from the preexisting vessels by migration and proliferation of endothelial cells, is an essential process during both intramembranous and endochondral bone formation, bone healing, and osseointegration of implants [2,3].

Vascular endothelial growth factor (VEGF) is a growth factor involved in many human physiologic processes such as angiogenesis [4]. VEGF is a key component of neovascularization and plays a crucial role in the restoration of vascular bone supply during the bone healing process $[4,5]$. Following implant placement in the bone and the initiation of the clotting process, the platelets release several cytokines and growth factors. These factors attract the inflammatory cells and mediate the chemotactic response. Several studies have shown the effectiveness of VEGFs on bone formation and bone tissue engineering models [6, 7]. Thus, any medication that inhibits VEGFs could potentially hinder bone healing and osseointegration. However, evidence remains scarce on osseointegration pharmacology and the impact of medication on osseointegration. Thus, a knowledgeable and expert clinician may expose an individual to some consequences or harm, due to the absence of any systematically developed, evidence-based guidelines. In order to respect the duty of care, assure quality of care, and meet the demands of thirdparty agencies and regulatory bodies, any possible harm or side effects or unexpected therapeutic failures should be reported, investigated, and assessed rapidly.

\section{Case Report}

Following CARE guidelines for case reports [8], this clinical report presents the case of an atypical implant failure that occurred during a clinical trial conducted at Université de 
Montréal, Oral Health and Rehabilitation Research Unit. The study was approved by the Université de Montréal Ethics Board, and informed consent was obtained from all participants. The results of this trial concerning the immediate loading of a two-implant unsplinted mandibular overdenture and the details of clinical procedures have been published previously [9]. In brief, following standard prosthodontic and surgical procedures, all study participants received a new set of maxillary and mandibular complete prosthesis (before the surgical phase) and three threaded implants (OsseoSpeed ${ }^{\mathrm{TM}}$, Dentsply Implants, Mölndal, Sweden) using an immediate-loading (within 24 hours of surgery) protocol on two of the three implants.

The connection of right- and left-side implants and prostheses was established via unsplinted abutments (Locator ${ }^{\circledR}$ abutment, ZEST Anchors L.L.C., Escondido, CA, USA). The midline implant was unloaded for within-patient comparison on peri-implant bone crest height and implant stability. The opposing maxillary dentition for all patients was rehabilitated with a conventional removable complete denture. Of the 18 participants, one participant lost the left implant because of parafunctional habit. Another patient who experienced implant loss (female, 76 years old), and who is the subject of this case report, lost both loaded implants. Implant loss occurred within 6 weeks of immediate loading. The medical and dental history and panoramic and cephalometric preoperative radiographs (see Figures 1(a) and 1(b)) as well as clinical examinations had not revealed any contraindication for insertion of implants and immediate-loading protocol. The patient had a knifeedge mandibular edentulous ridge with an anterior bone height of $13 \mathrm{~mm}$ and bone width at a midheight of $7 \mathrm{~mm}$. For both implants, primary stability was achieved and initial torque values were all above $50 \mathrm{Ncm}$ (see Figures 2(a) and 2(b)). After implant failure (Figure 3), the patient's medical history was reviewed again, and at that time, the patient informed the research team that she had been taking an intraocular injection of Ranibizumab (Lucentis ${ }^{\circledR}$, Genentech USA Inc., South San Francisco, CA, USA) every two months for about a year, for the treatment of age-related macular degeneration (AMD). She specified that 20 days before implant placement, she had received an injection. The mandibular overdenture of the patient was converted into a single-implant overdenture by placing a Locator ${ }^{\circledR}$ anchor system on the midline implant. To date, i.e., 7 years after implant placement, the patient has not reported any complication with her prosthesis.

\section{Discussion}

This clinical report suggests a possible impact of VEGF inhibitors on implant osseointegration. VEGF inhibitors such as Ranibizumab are used routinely for the treatment of age-related macular degeneration (AMD) $[10,11]$. AMD is a disease associated with aging. It is a leading cause of adult irreversible blindness in developed countries and the third cause of worldwide blindness [12-14]. AMD has two major types: nonexudative or atrophic or "dry" AMD and neovascular or exudative or "wet" AMD. The wet form of AMD is characterized by choroidal neovascularization, the growth of abnormal blood vessels from the choroid underneath the macula [15]. Ranibizumab binds to VEGFs and inhibits VEGF-dependent angiogenesis and decreases vascular permeability [16]. As a consequence, this medication has the potential to have a negative impact on osseointegration. However, the extent to which an intravitreal injection of Ranibizumab might have systemic side effects remains unclear and should be addressed in future studies.

Despite distinctive clinical relevance, knowledge about the potential effect of inhibiting VEGF-dependent angiogenesis in dental implant osseointegration is limited. Mair et al. studied the impact of TNP-470, an inhibitor of angiogenesis, on the peri-implant bone formation and osseointegration [17]. Their results showed a negative effect and highlighted the need to investigate the effect of VEGF inhibitors on osseointegration. The findings of our recent in vivo animal study supported this clinical report hypothesis. In this study, the effects of VEGF inhibitors on bone healing and implant osseointegration were examined in the rat tibia [18]. The bone-implant contact percentage, angiogenesis, and bone defect volume were assessed by the use of histology, histomorphometry, and micro-CT analysis. The results showed that in rats that received anti-VEGF neutralizing antibody or Ranibizumab, bone-implant contact percentage was lower than in the control group, at the level of statistical significance. Furthermore, this treatment inhibited the formation of new blood vessels and diminished the blood vessel density. The size of the bone defect was significantly larger in the anti-VEGF group.

On the contrary, the potential impact of immediate loading on implant failure should not be ignored, especially since both immediately loaded implants failed while the midline control implant healed uneventfully. It was initially recommended, in the conventional oral implant-loading protocol for rehabilitation with a mandibular overdenture, that loading of the implant should occur after a minimum of 3 months of healing after implant placement in the mandible, to let the implant osseointegrate with the surrounding bone [19]. In recent years, with the development of new roughened implant surfaces, the literature has recommended immediate- or early-loading protocols in the mandible to improve the quality of life of edentate patients [20]. A recent meta-analysis on this topic showed similar implant success rates for immediate- or early-loading protocols when compared to the conventional loading protocol for mandibular implant overdentures [21]. In addition, the implant short-term success rate has been reported to not be associated with the number of implants or the design of suprastructure (splinted versus freestanding) [22]. Nevertheless, immediate exposure of the two implants to masticatory forces could be a potential cause for the bone resorption surrounding both implants. Therefore, we can conclude that the peri-implant bone submitted to an immediate loading might have been more susceptible to being affected by residual concentrations of Ranibizumab. In fact, as previously highlighted by Esposito et al. [23], it is not possible to predict implant failures. A clinical report contributes to scientific development in the preliminary stages 


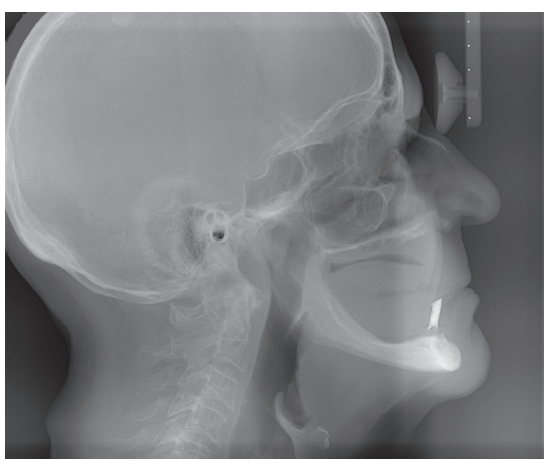

(a)

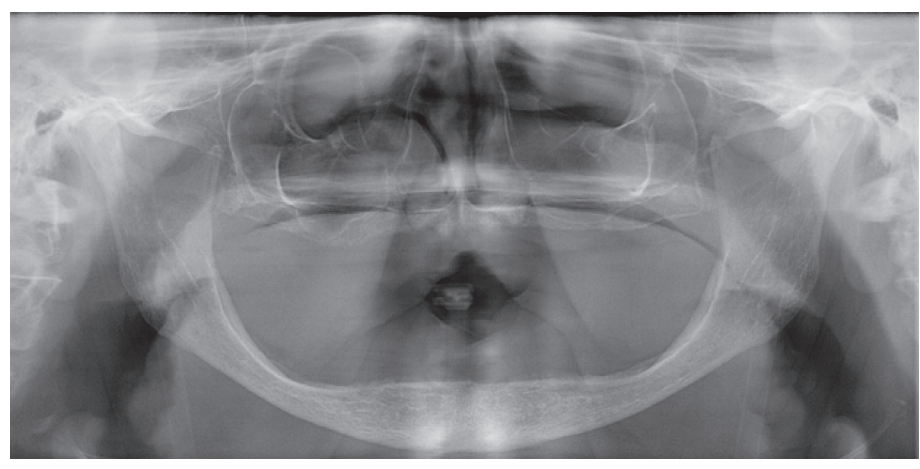

(b)

Figure 1: (a) Preoperative cephalometric radiograph. (b) Preoperative panoramic radiograph.

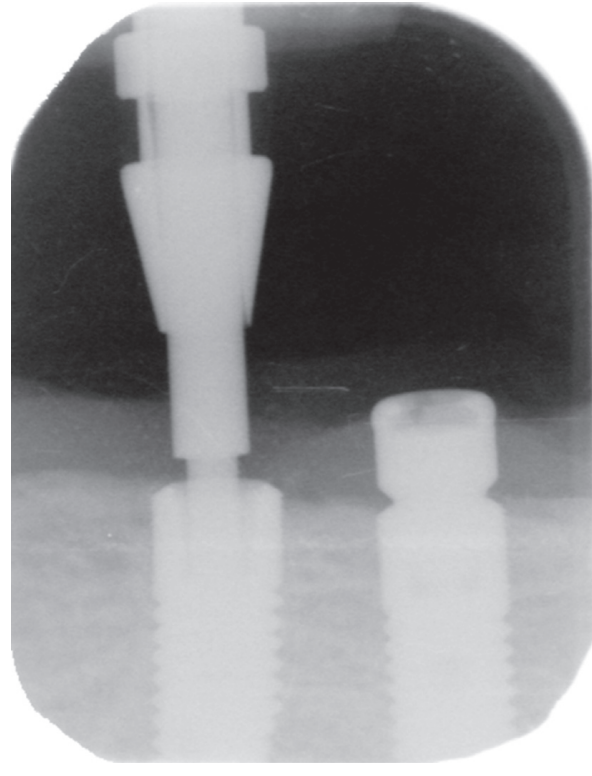

(a)

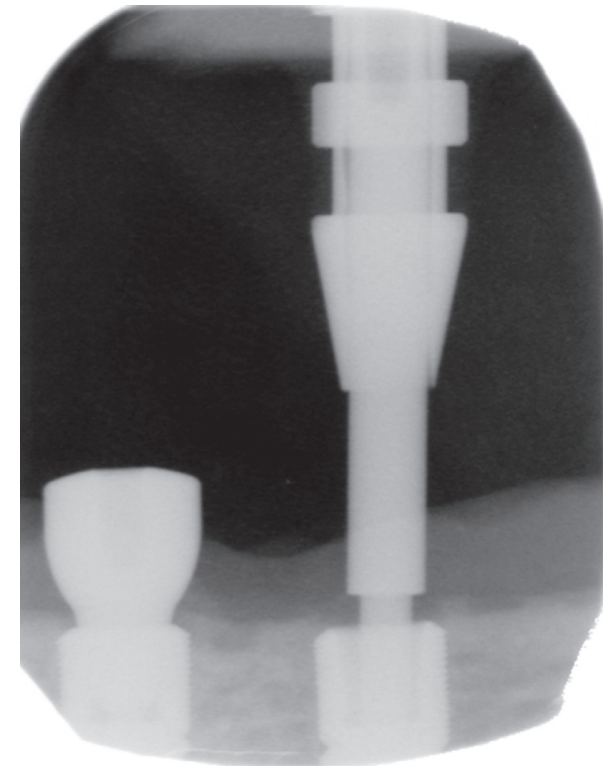

(b)

FIgURE 2: Standardized periapical radiograph taken immediately after (a) implant placement \#43 and (b) implant placement \#33.

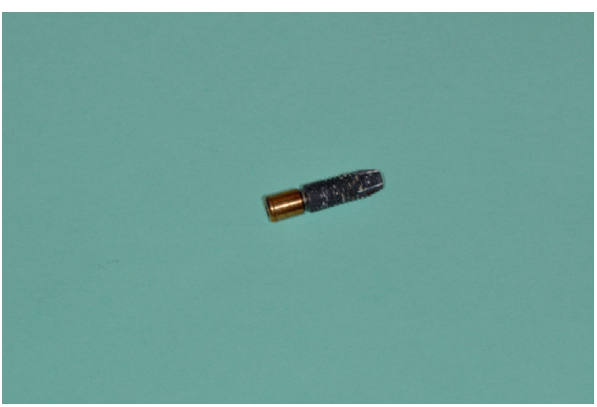

Figure 3: Implant loss.

of an investigation since it suggests hypotheses, which may be tested systematically from bedside to the bench and then, from laboratory experiments to large clinical trials, and finally to clinical guidelines. Further investigation on osseointegration and medication via collaborative research and merging data from several sources is recommended.

\section{Conclusion}

Implants may fail for different reasons that are not always easy to determine. The intake of Ranibizumab, a commonly used VEGF inhibitor, may introduce a risk for implant success due to its negative effects on bone formation and osseointegration. Data from large clinical cohorts are needed for further investigation on this topic.

\section{Conflicts of Interest}

The authors declare no potential conflicts of interest with respect to the publication of this article.

\section{Acknowledgments}

The study was supported by Dr. Elham Emami's ClinicianScientist Award at the Canadian Institutes of Health Research (CIHR) and Fonds de recherche en Santé du Québec 
(FRSQ). The main clinical trial received partial financial support from DENTSPLY Implants, and the in vivo study received financial support from the International Team for Implantology. We acknowledge the invaluable collaboration of Dr. Tamimi's research group at McGill University for the in vivo analysis, which allowed us deeper insight for this case report.

\section{References}

[1] "Oral implantology. glossary of implant terms," Journal of Oral Implantology, no. 1, pp. 2-14, vol. 33, 2007.

[2] M. I. Santos and R. L. Reis, "Vascularization in bone tissue engineering: physiology, current strategies, major hurdles and future challenges," Macromolecular Bioscience, vol. 10, no. 1, pp. 12-27, 2010.

[3] J. E. Davies, "Understanding peri-implant endosseous healing," Journal of Dental Education, vol. 67, no. 8, pp. 932-949, 2003.

[4] B. Beamer, C. Hettrich, and J. Lane, "Vascular endothelial growth factor: an essential component of angiogenesis and fracture healing," HSS Journal, vol. 6, no. 1, pp. 85-94, 2010.

[5] D. Ribatti, "The crucial role of vascular permeability factor/ vascular endothelial growth factor in angiogenesis: a historical review," British Journal of Haematology, vol. 128, no. 3, pp. 303-309, 2005.

[6] D. Gothard, E. L. Smith, J. M. Kanczler et al., "Tissue engineered bone using select growth factors: a comprehensive review of animal studies and clinical translation studies in man," European Cells and Materials, vol. 28, pp. 166-208, 2014.

[7] K. Hu and B. R. Olsen, "Osteoblast-derived VEGF regulates osteoblast differentiation and bone formation during bone repair," Journal of Clinical Investigation, vol. 126, no. 2, pp. 509-526, 2016.

[8] D. S. Riley, M. S. Barber, G. S. Kienle et al., "CARE guidelines for case reports: explanation and elaboration document," Journal of Clinical Epidemiology, vol. 89, pp. 218-235, 2017.

[9] E. Emami, D. Cerutti-Kopplin, M. Menassa et al., "Does immediate loading affect clinical and patient-centered outcomes of mandibular 2-unsplinted-implant overdenture? A 2year within-case analysis," Journal of Dentistry, vol. 50, pp. 30-36, 2016.

[10] F. Lu and R. A. Adelman, "Are intravitreal bevacizumab and ranibizumab effective in a rat model of choroidal neovascularization?" Graefe's Archive for Clinical and Experimental Ophthalmology, vol. 247, no. 2, pp. 171-177, 2009.

[11] P. J. Rosenfeld, D. M. Brown, J. S. Heier et al., "Ranibizumab for neovascular age-related macular degeneration," New England Journal of Medicine, vol. 355, no. 14, pp. 1419-1431, 2006.

[12] R. Klein, T. Peto, A. Bird, and M. R. Vannewkirk, "The epidemiology of age-related macular degeneration," American Journal of Ophthalmology, vol. 137, no. 3, pp. 486-495, 2004.

[13] N. Congdon, B. O'Colmain, C. C. Klaver et al., "Causes and prevalence of visual impairment among adults in the United States," Archives of Ophthalmology, vol. 122, no. 4, pp. 477485, 2004.

[14] C. A. Augood, J. R. Vingerling, P. T. V. M. de Jong et al., "Prevalence of age-related maculopathy in older Europeans," Archives of Ophthalmology, vol. 124, no. 4, pp. 529-535, 2006.
[15] J. Z. Nowak, "Age-related macular degeneration (AMD): pathogenesis and therapy," Pharmacological Reports, vol. 58, no. 3, pp. 353-363, 2006.

[16] S. S. Vedula and M. G. Krzystolik, "Antiangiogenic therapy with anti-vascular endothelial growth factor modalities for neovascular age-related macular degeneration," The Cochrane Database of Systematic Reviews, vol. 2, 2008.

[17] B. Mair, G. Fuerst, P. Kubitzky et al., "The anti-angiogenic substance TNP-470 impairs peri-implant bone formation: a pilot study in the rabbit metaphysis model," Clinical Oral Implants Research, vol. 18, no. 3, pp. 370-375, 2007.

[18] A. E. Al Subaie, H. Eimar, M.-N. Abdallah et al., "Anti-VEGFs hinder bone healing and implant osseointegration in rat tibiae," Journal of Clinical Periodontology, vol. 42, no. 7 , pp. 688-696, 2015.

[19] P. I. Brånemark, B. O. Hansson, R. Adell et al., "Osseointegrated implants in the treatment of the edentulous jaw. Experience from a 10-year period," Scandinavian Journal of Plastic and Reconstructive Surgery. Supplementum, vol. 16, pp. 1-132, 1977.

[20] F. Borges Tde, F. A. Mendes, T. R. de Oliveira, V. L. Gomes, C. J. do Prado, and F. D. das Neves, "Mandibular overdentures with immediate loading: satisfaction and quality of life," International Journal of Prosthodontics, vol. 24, no. 6, pp. 534-539, 2011.

[21] N. Alsabeeha, M. Atieh, and A. G. Payne, "Loading protocols for mandibular implant overdentures: a systematic review with meta-analysis," Clinical Implant Dentistry and Related Research, vol. 12, no. 1, pp. e28-e38, 2010.

[22] M. Esposito, M. G. Grusovin, H. Maghaireh, and H. V. Worthington, "Interventions for replacing missing teeth: different times for loading dental implants," The Cochrane Database of Systematic Reviews, Article ID CD003878, vol. 3, 2013.

[23] M. Esposito, M. G. Grusovin, H. Achille, P. Coulthard, and H. V. Worthington, "Interventions for replacing missing teeth: different times for loading dental implants," The Cochrane Database of Systematic Reviews, Article ID CD003878, vol. 1, 2009. 